\title{
Absorbing Boundary Conditions for Difference Approximations to the Multi-Dimensional Wave Equation
}

\author{
By Robert L. Higdon
}

\begin{abstract}
We consider the problem of constructing absorbing boundary conditions for the multi-dimensional wave equation. Here we work directly with a difference approximation to the equation, rather than first finding analytical boundary conditions and then discretizing the analytical conditions. This approach yields some simple and effective discrete conditions.

These discrete conditions are consistent with analytical conditions that are perfectly absorbing at certain nonzero angles of incidence. This fact leads to a simple and general canonical form for analytical absorbing boundary conditions. The use of this form has theoretical and practical advantages.
\end{abstract}

1. Introduction. Consider the wave equation

$$
u_{t t}=u_{x x}+u_{y y}
$$

for $x>0, y \in R, t>0$. Here $u$ is a function of $x, y$, and $t$. The units have been normalized so that the wave speed is 1 . Let $\Omega$ denote the spatial domain $\{(x, y)$ : $x>0, y \in R\}$.

Our goal is to find boundary conditions that cause wave motions from the interior of $\Omega$ to pass through the boundary without being reflected. In general, it is not possible to find practical boundary conditions that do this perfectly (see, e.g., [3], [7]). Instead, one wishes to find conditions that reduce the amount of reflection as much as possible.

Boundary conditions of this type are desirable in a number of physical problems (see, e.g., [1], [4], and references cited therein). In such situations, an open, artificial boundary has been introduced in order to limit a large or unbounded domain so that a numerical computation of the solution can be made feasible. One wants the solution to behave as though the computational boundary were not present. In particular, outgoing wave motions should pass through the boundary without being reflected.

Substantial work has been performed on problems of this type. See, e.g., Bayliss and Turkel [1] and Engquist and Majda [3], [4]. By and large, prior work has concentrated on finding analytical boundary conditions for differential equations

Received October 12, 1984; revised August 7, 1985.

1980 Mathematics Subject Classification. Primary 35L05, 65M99, 65N99.

Key words and phrases. Absorbing boundary conditions, wave equation, initial-boundary value problems. 
rather than discrete boundary conditions for difference approximations. Some exceptions are the analyses of the one-dimensional wave equation by Halpern [7] and by Engquist and Majda in Section 5 of [4].

In the present paper we work directly with a difference approximation to (1.1), rather than first finding analytical boundary conditions and then discretizing the analytical conditions. This approach yields some simple and effective discrete conditions.

These conditions turn out to be discretizations of some analytical boundary conditions that are perfectly absorbing for waves traveling at certain nonzero angles of incidence. This contrasts with the boundary conditions in [3] and [4], which work best at normal incidence. We present some numerical tests that indicate the value of the present approach.

We also describe a process by which the above conditions can be generalized to arbitrary angles of incidence. This procedure leads to a simple and fairly general characterization of analytical absorbing boundary conditions. This characterization includes those in [3], [4] and is related to those in [1].

The outline of the paper is as follows. In Section 2 we make some preliminary comments about dispersion relations and group velocity. In Section 3 we define some notation, make a precise formulation of the problem, and make some comments about stability. In Sections 4 and 5 we present the discrete boundary conditions mentioned above. In Section 6 we present the results of the numerical computations. In Section 7 we describe the generalization to arbitrary angles of incidence. In Section 8 we discuss some practical aspects of the generalization. In Section 9 we describe a factorization of absorbing boundary conditions that yields the general characterization mentioned above.

2. Dispersion Relations and Group Velocity. Here we state some facts about dispersion relations and group velocity that will be used in later sections.

When a wave form

$$
e^{i \sigma x+i \omega y+i \xi t}
$$

is inserted into (1.1), the result is the "dispersion relation"

$$
\xi^{2}=\sigma^{2}+\omega^{2} .
$$

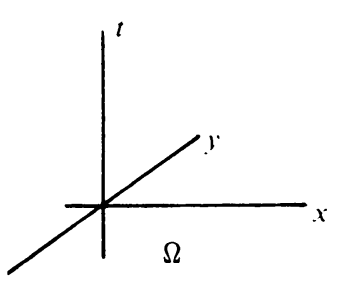

(a) Space-time domain

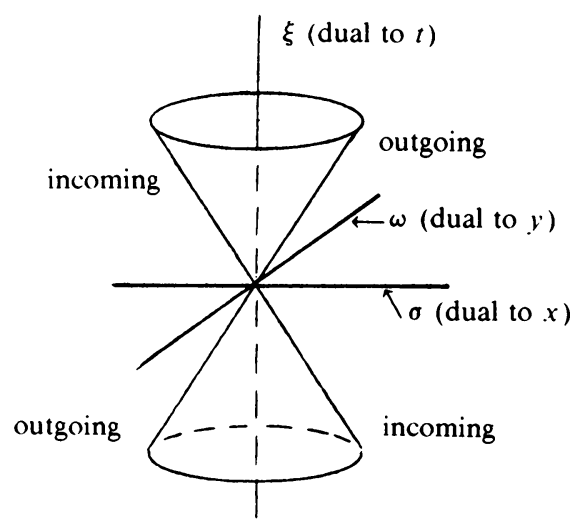

(b) Frequency domain 
The graph of (2.2) is given in Figure 2.1(b). For general wave motions, one can show that wave packets of neighboring frequencies travel at the group velocity

$$
\left(-\frac{\partial \xi}{\partial \sigma},-\frac{\partial \xi}{\partial \omega}\right)
$$

(see, e.g., Whitham [16], Trefethen [13]). In the present case the group velocity coincides with the phase velocity $(-\sigma / \xi,-\omega / \xi)$. The gradient $(2.3)$ enables one to associate various portions of the cone in Figure 2.1(b) with motion into or out of the spatial domain $\Omega$.

Next, consider difference approximations. Introduce a rectangular grid with mesh spacings $\Delta x, \Delta y, \Delta t$ in the $x, y, t$ directions, respectively, and let $u_{j, m}^{n}$ denote the approximation to $u(j \Delta x, m \Delta y, n \Delta t)$. Throughout this paper we will consider the standard second-order centered approximation

$$
\begin{aligned}
\frac{u_{j, m}^{n+1}-2 u_{j, m}^{n}+u_{j, m}^{n-1}}{(\Delta t)^{2}}= & \frac{u_{j+1, m}^{n}-2 u_{j, m}^{n}+u_{j-1, m}^{n}}{(\Delta x)^{2}} \\
& +\frac{u_{j, m+1}^{n}-2 u_{j, m}^{n}+u_{j, m-1}^{n}}{(\Delta y)^{2}} .
\end{aligned}
$$

When (2.1) is inserted into (2.4), the result is

$$
\left(\sin \frac{\xi \Delta t}{2}\right)^{2}=\left(\frac{\Delta t}{\Delta x}\right)^{2}\left(\sin \frac{\sigma \Delta x}{2}\right)^{2}+\left(\frac{\Delta t}{\Delta y}\right)^{2}\left(\sin \frac{\omega \Delta y}{2}\right)^{2}
$$

This is the dispersion relation for the difference scheme (2.4). The graph of (2.5) is given in Figure 2.2.

The wave form (2.1) can be written as

$$
\left(e^{i \sigma \Delta x}\right)^{j}\left(e^{i \omega \Delta y}\right)^{m}\left(e^{i \xi \Delta t}\right)^{n}
$$

for points $(j \Delta x, m \Delta y, n \Delta t)$ on the grid. This means that the quantities $\sigma \Delta x, \omega \Delta y$, $\xi \Delta t$ can be confined to the interval $[-\pi, \pi]$. If each of these quantities is small, then the wave (2.6) is resolved well by the grid, and (2.5) approximates (2.2). However, for larger frequencies the group velocities and phase velocities differ, and substantial dispersion occurs. For example, the group velocity is zero if $\sigma \Delta x= \pm \pi$ and $\omega=0$ (see (2.3) and Figure 2.2). An extensive discussion of dispersive effects in finitedifference schemes is given by Trefethen [12].

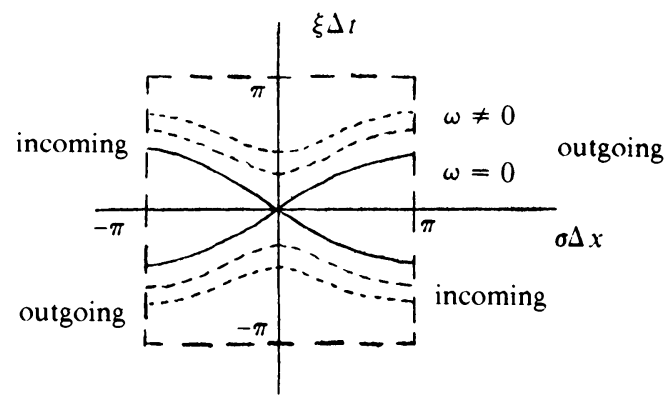

FiguRE 2.2

Graph of the dispersion relation for the difference approximation. (Cross sections for fixed $\omega$.) 
The "incoming" and "outgoing" portions of the solution are indicated in Figure 2.2 (cf. (2.3)).

3. General Formulation of the Problem. Here we define some notation and describe some general properties of the boundary conditions that are desired here.

The wave form (2.6) can be written as

$$
\kappa^{j} e^{i \omega y} z^{n},
$$

where

$$
\kappa=e^{i \sigma \Delta x} \text { and } z=e^{i \xi \Delta t} .
$$

If $\sigma \Delta x=0$, then $\kappa=1$ and the wave is constant in $x$. If $\sigma \Delta x= \pm \pi$, then $\kappa=-1$ and the wave is a sawtooth in $x$. Analogous comments hold for $z$.

Let $K$ and $Z$ denote the shift operators with respect to $x$ and $t$, respectively, defined by

$$
K u_{j, m}^{n}=u_{j+1, m}^{n}, \quad Z u_{j, m}^{n}=u_{j, m}^{n+1} .
$$

We will consider boundary conditions of the form

$$
B\left(K, Z^{-1}\right) u_{0, m}^{n+1}=0,
$$

where $B$ is a polynomial in two variables having a nonzero constant term. The boundary condition (3.4) expresses the boundary value $u_{0, m}^{n+1}$ in terms of values at earlier times and/or in the interior of the spatial domain. The boundary conditions used later do not involve shifts in the $y$-direction.

In order to study the reflection properties of (3.4), consider a linear combination

$$
u_{j, m}^{n}=c_{1} \kappa_{1}^{j}\left(e^{i \omega \Delta y}\right)^{m} z^{n}+c_{2} \kappa_{2}^{j}\left(e^{i \omega \Delta y}\right)^{m} z^{n} .
$$

Here $\kappa_{1}=\exp \left(i \sigma_{1} \Delta x\right)$ and $\kappa_{2}=\exp \left(i \sigma_{2} \Delta x\right)$ are chosen so as to correspond to incoming and outgoing group velocity, respectively (see Figure 2.2). (Strictly speaking, one would consider wave packets formed by integrating with respect to $\omega$ and $\xi$. But the analysis amounts to studying single frequency pairs $(\omega, \xi)$.) When (3.5) is inserted into (3.4), the result is

$$
c_{1} B\left(\kappa_{1}, z^{-1}\right)+c_{2} B\left(\kappa_{2}, z^{-1}\right)=0,
$$

or

$$
c_{1}=-\frac{B\left(\kappa_{2}, z^{-1}\right)}{B\left(\kappa_{1}, z^{-1}\right)} c_{2}=R(\omega, z) c_{2},
$$

where $R(\omega, z)$ is the reflection coefficient. The notation $(\omega, z)$ is used here because $\kappa_{1}$ and $\kappa_{2}$ are functions of $\omega$ and $z$.

Our goals are the following:

(1) Choose the boundary operator $B$ so that $|R|$ is as small as possible. The ideal situation would be to have

$$
B\left(\kappa_{2}, z^{-1}\right)=0
$$

for all possible $z$ and corresponding $\kappa_{2}$. This means that the outgoing waves would satisfy the boundary condition exactly, so that the boundary condition could be regarded as a compatibility condition for outgoing radiation. However, this ideal cannot be attained exactly in practice. 
(2) Maintain stability. Roughly speaking, we will use the criterion

$$
B\left(\kappa, z^{-1}\right) \neq 0 \text { whenever }|z| \geqslant 1,|\kappa| \leqslant 1 \text {, }
$$

and $\kappa^{j} e^{i \omega y} z^{n}$ is a solution of the interior difference equation. (Here $\kappa$ and $z$ are complex numbers, and we are extending the definition of $\kappa$ and $z$ given in (3.2).) This criterion needs to be made more precise.

(a) Suppose that $|z|>1,|\kappa|<1$, and $\kappa^{j} e^{i \omega y} z^{n}$ is a solution of the difference equation. If this mode also satisfies the boundary condition, then one has a solution to the given problem that grows exponentially in the number of time steps. This constitutes an obvious instability, and condition (3.7) is therefore essential.

(b) Now let $|z| \rightarrow 1$, and suppose that $|\kappa| \rightarrow 1$ also. (These limits are taken from the domains $|z|>1,|\kappa|<1$.) One then has a purely oscillatory mode like those discussed earlier. It can be shown that a mode obtained by this limiting process must have a group velocity that points into the spatial domain. Thus $\kappa=\kappa_{1}$. See, e.g., [8] or [12]. In this case the criterion (3.7) says that incoming waves must not be allowed to satisfy the boundary condition by themselves. If this criterion were not satisfied, then waves could radiate inward from the boundary without any stimulation from outgoing waves or forcing functions in the boundary condition. This would be a form of instability.

Case (b) above does not cover the case of evanescent modes for which $|z|=1$ and $|\kappa|<1$. For such modes, the exponential decay in $x$ means that any undesirable effects would be confined to a neighborhood of the boundary $x=0$.

Extensive discussions of the above issues have been given by Trefethen [13].

Criteria like (3.7) have been used to prove stability results for first-order hyperbolic systems. See Gustafsson, Kreiss, and Sundström [5] and Michelson [11]. In the present case, we will regard (3.7) as a useful guideline for constructing and analyzing boundary conditions.

It is important to note that goals (1) and (2) above are partly contradictory. The trouble occurs at the zero frequency $\sigma \Delta x=\xi \Delta t=\omega \Delta y=0$, i.e., $\kappa=z=1$ and $\omega=0$. This is the point where the two branches of the dispersion relation cross (see Figure 2.2). The case $\kappa=z=1$ thus corresponds both to incoming and outgoing waves. If the boundary condition annihilates the outgoing mode at $\kappa=z=1$ (i.e., $B(1,1)=0$ ), then it also annihilates the incoming mode, and condition (3.7) is violated. On the other hand, if (3.7) is satisfied for $\kappa=z=1$, then at that point the reflection coefficient satisfies $|R|=1$. Thus, there is total reflection at the zero frequency and substantial reflection for low frequencies.

The label "generalized eigenvalue" is sometimes associated with situations where a boundary condition allows incoming waves for $|z|=1$, as in the present case (see, e.g., [8] or [13]). Trefethen [13] has shown that mild instabilities can be present in such situations. These take the form of waves radiating spontaneously into the spatial domain from the boundary. In Section 6 we present some numerical tests with low-frequency data which suggest that these effects can be substantial when the order of the zero in $B\left(\kappa_{1}, z^{-1}\right)$ exceeds two. However, for methods of order two or less, these effects are outweighed by the advantages of small reflection coefficients. Another test suggests that there is less of a problem with high-frequency data. The generalized eigenvalues thus do not prevent one from finding effective absorbing boundary conditions, and we seek $B(1,1)=0$. 
4. An Averaging Method. We now develop the first class of absorbing boundary conditions to be discussed in this paper. These conditions are defined by

$$
\left[I-\left(\frac{I+Z^{-1}}{2}\right)\left(\frac{I+K}{2}\right)\right]^{p} u_{0, m}^{n+1}=0 .
$$

Here $p$ can be any positive integer, and $K$ and $Z$ are the shift operators defined in (3.3). These boundary conditions have one-dimensional stencils but have strong multi-dimensional effects. The first-order version $(p=1)$ simplifies to

$$
\left[I-\frac{1}{3}\left(K+Z^{-1}+Z^{-1} K\right)\right] u_{0, m}^{n+1}=0
$$

or

$$
u_{0, m}^{n+1}=\frac{1}{3}\left(u_{1, m}^{n+1}+u_{0, m}^{n}+u_{1, m}^{n}\right),
$$

which suggests that the condition is approximately compatible with outgoing waves.

Here we derive and analyze (4.1) by working directly with properties of the difference scheme (2.4). The analysis will be based on the form given in (4.1) rather than any simplified forms.

A motivation for this form is given by the following. We seek a boundary condition,

$$
A\left(K, Z^{-1}\right) u_{0, m}^{n+1}=0,
$$

that satisfies constraints (1) and (2) mentioned in Section 3. (The notation $A\left(\kappa, z^{-1}\right)$ is used here to avoid conflict with the general discussion given in Section 3.) We also seek $A\left(\kappa, z^{-1}\right)=1$ whenever $\kappa=-1$ or $z=-1$. This would mean that the operator $A\left(K, Z^{-1}\right)$ is the identity operator for "parasitic" modes that are sawtooth in $x$ and/or $t$. This requirement is introduced in order to have an operator that can be applied to certain other hyperbolic problems where parasitic waves can be an issue. Roughly speaking, we will guarantee that the parasitic modes cannot accidentally satisfy the homogeneous boundary conditions and thereby generate instabilities.

The desired values of $A\left(\kappa, z^{-1}\right)$ are suggested by the labelling of various points in Figure 4.1(b). The other quadrants can be labelled similarly. The axes correspond to cross sections of the dispersion relation for fixed $\omega$. The center of the picture corresponds to $\kappa=z=1$, since $\kappa=\exp (i \sigma \Delta x)$ and $z=\exp (i \xi \Delta t)$. The edges correspond to $\kappa=-1$ and/or $z=-1$.

First consider

$$
P\left(\kappa, z^{-1}\right)=\left(\frac{1+z^{-1}}{2}\right)\left(\frac{1+\kappa}{2}\right) .
$$

Various values of $P\left(\kappa, z^{-1}\right)$ are suggested by Figure 4.1(a). If we then let

$$
A\left(\kappa, z^{-1}\right)=1-P\left(\kappa, z^{-1}\right)
$$

we get the desired values indicated in Figure 4.1(b). The corresponding difference operator $A\left(K, Z^{-1}\right)$ yields (4.1) for $p=1$. The higher-order method in (4.1) is obtained by using the operator $A\left(K, Z^{-1}\right)^{p}$.

It may appear that one could use $z$ in place of $z^{-1}$ and/or $\kappa^{-1}$ in place of $\kappa$. However, the goal here is to produce practical boundary conditions. Relative to any given boundary point, we need to have shifts backward in time and forward in space, so that the boundary condition uses values of the solution that already have been computed. 


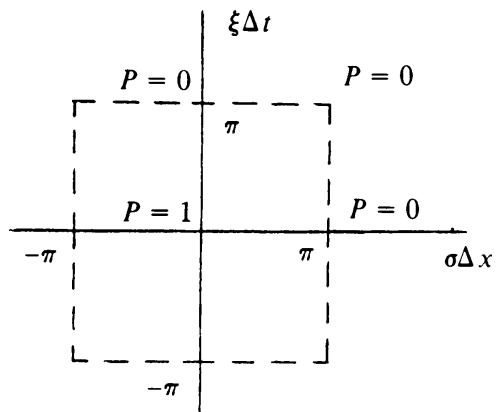

(a) Values of $P\left(\kappa, z^{-1}\right)$

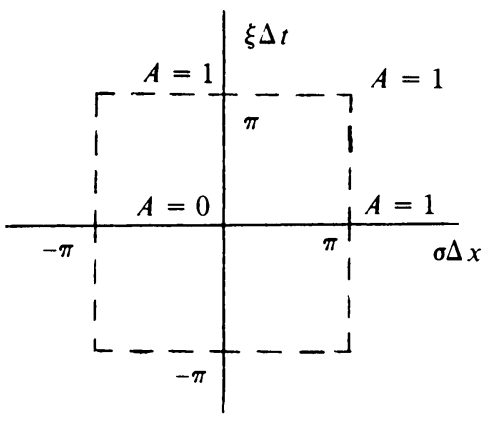

(b) Values of $A\left(\kappa, z^{-1}\right)$

FigURE 4.1

Proposition 4.1. The method (4.1) satisfies the stability criterion (3.7),

$$
A\left(\kappa, z^{-1}\right)^{p} \neq 0 \text { for }|z| \geqslant 1,|\kappa| \leqslant 1,
$$

with the exception that $A(1,1)=0$.

Proof. The $z$-factor in $P\left(\kappa, z^{-1}\right)$ in (4.2) satisfies

$$
\left|\frac{1+z^{-1}}{2}\right|<1
$$

for $|z| \geqslant 1$, except when $z=1$. (Consider vectors in the complex plane extending from -1 to $z^{-1}$.) A similar argument holds for the $\kappa$-factor. Thus $\left|P\left(\kappa, z^{-1}\right)\right|<1$ for $|z| \geqslant 1,|\kappa| \leqslant 1$ except when $z=\kappa=1$. A comparison with (4.3) completes the proof.

The exceptional case $A(1,1)=0$ means that there is a generalized eigenvalue corresponding to the zero frequency (see Section 3 ).

Next consider the reflection coefficients for (4.1). According to (3.6) and (4.1)-(4.3), these are given by

$$
R_{p}(\omega, z)=-\frac{A\left(\kappa_{2}, z^{-1}\right)^{p}}{A\left(\kappa_{1}, z^{-1}\right)^{p}}=-\left(-R_{1}(\omega, z)\right)^{p}
$$

Proposition 4.2. If $|z|=\left|\kappa_{1}\right|=\left|\kappa_{2}\right|=1$ and $z \neq 1$, then the reflection coefficient for the case $p=1$ satisfies $\left|R_{1}(\omega, z)\right|<1$, except when $\kappa_{1}=\kappa_{2}=1$ or $\kappa_{1}=\kappa_{2}=-1$.

This proposition applies to all oscillatory waves admitted by the interior difference scheme, not just those that are resolved well by the grid.

The exceptional case is of little consequence, since the $x$-component of the group velocity is zero for such modes (see Figure 2.2, and note that the hypothesis $z \neq 1$ implies $\omega \neq 0$ ). These modes do not propagate into the interior.

For all other cases, the proposition and (4.4) imply $R_{p}(\omega, z) \rightarrow 0$ as $p \rightarrow \infty$. However, the generalized eigenvalue corresponding to $z=\kappa=1$ places a limit on the value of $p$ that can be used in practice (see Section 6).

Proof of Proposition 4.2. The factors in the product $P\left(\kappa, z^{-1}\right)$ in (4.2) have the geometric representations given in Figure 4.2 (consider $z^{-1}-(-1)$, etc.). We will use these to compare $P\left(\kappa_{1}, z^{-1}\right)$ (incoming) and $P\left(\kappa_{2}, z^{-1}\right)$ (outgoing) and then use (4.3) u compare $A\left(\kappa_{1}, z^{-1}\right)$ and $A\left(\kappa_{2}, z^{-1}\right)$. 


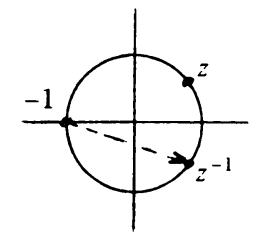

z-plane

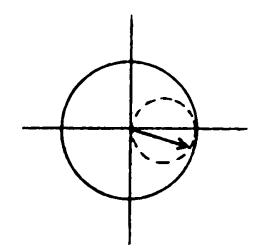

Representation of $\left(1+z^{-1}\right) / 2$

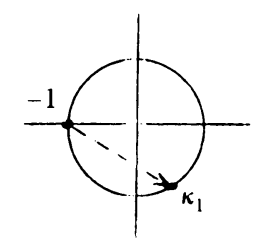

$\kappa_{1}$ (incoming)

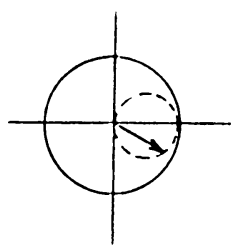

Representation of $\left(1+\kappa_{1}\right) / 2$

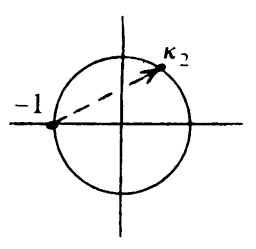

$\kappa_{2}$ (outgoing)

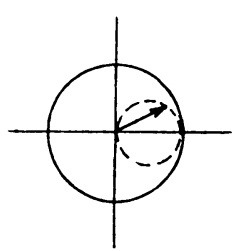

Representation of $\left(1+\kappa_{2}\right) / 2$

FIGURE 4.2

Here, $z=\exp (i \xi \Delta t)$ and $\kappa_{j}=\exp \left(i \sigma_{j} \Delta x\right)$. Figure 2.2 implies that $\xi \Delta t$ and $\sigma \Delta x$ have opposite signs for incoming modes and the same sign for outgoing modes. Thus, $\arg z^{-1}$ and $\arg \kappa_{1}$ have the same sign, and $\arg z^{-1}$ and $\arg \kappa_{2}$ have opposite signs (see Figure 4.2). Furthermore, the symmetry of the dispersion relation (2.5) implies $\arg \kappa_{2}=-\arg \kappa_{1}$. It follows that the products

$$
P\left(\kappa_{1}, z^{-1}\right)=\left(\frac{1+z^{-1}}{2}\right)\left(\frac{1+\kappa_{1}}{2}\right), \quad P\left(\kappa_{2}, z^{-1}\right)=\left(\frac{1+z^{-1}}{2}\right)\left(\frac{1+\kappa_{2}}{2}\right),
$$

have the same moduli. However, in the second case, there is partial cancellation of arguments whenever $\kappa_{1} \neq \kappa_{2}$, but not in the first. Thus, $P\left(\kappa_{2}, z^{-1}\right)$ is closer to 1 than is $P\left(\kappa_{1}, z^{-1}\right)$, whenever $\kappa_{1} \neq \kappa_{2}$. A comparison with (4.3) yields the desired conclusion.

The next proposition applies only to those oscillatory waves that are resolved well by the grid.

Proposition 4.3. The reflection coefficient $R_{p}(\omega, z)$ for (4.1) satisfies

$$
R_{p}(\omega, z)=-\left(\frac{\lambda-\cos \theta}{\lambda+\cos \theta}\right)^{p}+O(\xi \Delta t) .
$$

Here, $\lambda=\Delta t / \Delta x$, and $\theta$ is the angle of incidence measured relative to normal incidence.

The Courant-Friedrichs-Lewy condition for the interior difference scheme (2.4) requires $\lambda \leqslant 2^{-1 / 2}$ when $\Delta x=\Delta y$. If $\lambda$ has the maximum permissible value, then the angle of best absorption is 45 degrees.

Proof of Proposition 4.3. The reflection coefficient is

$$
R_{p}(\omega, z)=-\left[\frac{1-\frac{1}{2}\left(1+z^{-1}\right) \frac{1}{2}\left(1+\kappa_{2}\right)}{1-\frac{1}{2}\left(1+z^{-1}\right) \frac{1}{2}\left(1+\kappa_{1}\right)}\right]^{p}
$$

(See (4.3), (4.4).) Apply Taylor expansions to $z=\exp (i \xi \Delta t)$ and $\kappa_{j}=\exp \left(i \sigma_{j} \Delta x\right)$ to get

$$
R_{p}(\omega, z)=-\left(\frac{\sigma_{2}-\lambda \xi}{\sigma_{1}-\lambda \xi}\right)^{p}+O(\xi \Delta t)
$$




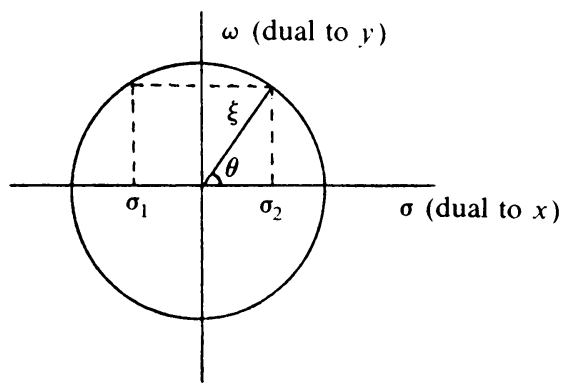

FIGURE 4.3

Cross section of Figure 2.1(b) for fixed $\xi>0$.

The dispersion relation (2.5) and the formula (2.3) for group velocity imply that, to leading order, $\sigma_{2} / \xi \approx \cos \theta$ and $\sigma_{1} / \xi \approx-\cos \theta$. Thus (4.6) implies (4.5).

An alternate approach to the last step is to observe that (2.5) implies $\xi^{2} \approx \sigma^{2}+\omega^{2}$ for waves that are resolved well by the grid. Thus, for leading-order effects, we may think in terms of the dispersion relation for the differential equation and its graph in Figure 2.1(b). Figure 4.3 shows a cross section of Figure 2.1(b) for fixed $\xi>0$. A comparison of $(2.3)$ with the cross section yields the conclusions given above. The case $\xi<0$ is treated in a similar manner.

Proposition 4.3 implies that the reflection is essentially zero when $\cos \theta=\lambda$ and $|\xi \Delta t|$ is small. This can be described in the following way. If

$$
\left|\arg \left(\frac{1+z^{-1}}{2}\right)\right|=\left|\arg \left(\frac{1+\kappa_{2}}{2}\right)\right|,
$$

then $P\left(\kappa_{2}, z^{-1}\right)$ is real (see Figure 4.2). If, in addition, $z$ and $\kappa_{2}$ are close to 1 , then $P\left(\kappa_{2}, z^{-1}\right)$ is much closer to 1 than is $P\left(\kappa_{1}, z^{-1}\right)$, and the reflection is essentially zero. But (4.7) is equivalent to $\arg z^{-1}=\arg \kappa_{2}$, or $\xi \Delta t=\sigma_{2} \Delta x$. Thus, in this case, $\cos \theta \approx \lambda$.

Proposition 4.4. The method (4.1) is consistent with the analytical boundary condition

$$
\left(\lambda \frac{\partial}{\partial t}-\frac{\partial}{\partial x}\right)^{p} u=0
$$

Proof. For example, (4.1) can be written as

$$
\left[\frac{\Delta t}{\Delta x}\left(\frac{I-Z^{-1}}{\Delta t}\right)-\left(\frac{I+Z^{-1}}{2}\right)\left(\frac{K-I}{\Delta x}\right)\right]^{p} u_{0, m}^{n+1}=0 .
$$

Let $\Delta x \rightarrow 0$ with the ratio $\lambda=\Delta t / \Delta x$ fixed.

The coefficient of $\partial / \partial t$ in (4.8) is the cosine of the angle of best absorption. This suggests a generalization that will be discussed in Sections 7, 8, and 9.

We conclude by outlining another feature of the operator $A\left(K, Z^{-1}\right)$ defined in (4.3). Simple modifications of this operator could be composed with boundary conditions in other hyperbolic problems in order to suppress undesirable parasitic waves. For example, to suppress a parasite corresponding to $\kappa=-1, z=1$, use the operator defined by

$$
1-\left(\frac{1+z^{-1}}{2}\right)\left(\frac{1-\kappa}{2}\right)
$$


This function is zero for $\kappa=-1, z=1$. However, the function is equal to 1 for $\kappa=1$ and for $\kappa=z=-1$, so that the operator is turned off away from the mode in question. Similar variations can be used for parasitic modes at $\kappa=1$ and $z=-1$ or $\kappa=z=-1$.

5. Space-Time Extrapolation. In this section we consider the "space-time extrapolation" boundary conditions

$$
\left(I-Z^{-1} K\right)^{p} u_{0, m}^{n+1}=0 .
$$

Here, $p$ is a positive integer. Some examples are the following:

$$
\begin{array}{ll}
p=1: & u_{0, m}^{n+1}=u_{1, m}^{n}, \\
p=2: & u_{0, m}^{n+1}=2 u_{1, m}^{n}-u_{2, m}^{n-1} .
\end{array}
$$

The stencils are diagonal patterns in the $(x, t)$-plane.

Some properties of difference operators and polynomial interpolation imply that these boundary conditions are true polynomial extrapolations. For example, when $p=2$, the boundary condition has the effect of fitting a linear function to the values $u_{1, m}^{n}$ and $u_{2, m}^{n-1}$ and extrapolating to the boundary. The case $p=2$ can thus be regarded as a "linear" space-time extrapolation, but we will refer to it as "second order" in order to be consistent with other terminology used in this paper.

Space-time extrapolation has been shown to be a stable outflow boundary condition for certain common difference approximations to first-order hyperbolic systems. See, e.g., Gustafsson, Kreiss, and Sundström [5], Beam, Warming, and Yee [2], and Gustafsson and Oliger [6]. Here we consider its application to the wave equation.

First, consider stability. Let $S_{p}\left(K, Z^{-1}\right)$ denote the operator $\left(I-Z^{-1} K\right)^{p}$ used in (5.1).

Proposition 5.1. Space-time extrapolation of order $p$ satisfies the stability criterion (3.7), with the exception that $S_{p}(1,1)=0$.

Proof. First suppose $|z|>1$ and $|\kappa|<1$. Then $\left|z^{-1} \kappa\right|<1$, and $1-z^{-1} \kappa \neq 0$.

Next, consider the limit $|z| \rightarrow 1$. If in this limit $|\kappa|<1$, then $\left|z^{-1} \kappa\right|<1$ as before. Otherwise, we have $|\kappa|=1$ in the limit. According to remarks made after (3.7), this limiting $\kappa$ must be $\kappa_{1}$ (incoming) instead of $\kappa_{2}$ (outgoing). Thus we need to show $1-z^{-1} \kappa_{1} \neq 0$, or $\xi \Delta t \neq \sigma_{1} \Delta x$. But for incoming modes, $\xi \Delta t$ and $\sigma \Delta x$ have opposite signs except when $\xi \Delta t=\sigma \Delta x=\omega \Delta y=0$ (see Figures 2.2 and 5.1). Also see Figures 4.2 and 5.2.

Next, consider the reflection coefficients for the boundary conditions (5.1). These are given by

$$
R_{p}^{S T}(\omega, z)=-\frac{\left(1-z^{-1} \kappa_{2}\right)^{p}}{\left(1-z^{-1} \kappa_{1}\right)^{p}}=-\left(\frac{z-\kappa_{2}}{z-\kappa_{1}}\right)^{p} .
$$

Proposition 5.2. Suppose $|z|=\left|\kappa_{1}\right|=\left|\kappa_{2}\right|=1$ and $z \neq 1$. Then

$$
\left|R_{p}^{S T}(\omega, z)\right|<1 \text {, }
$$

except when $\kappa_{1}=\kappa_{2}=1$ or $\kappa_{1}=\kappa_{2}=-1$.

As in Proposition 4.2, the exceptional case is of little significance. For all other cases, $R_{p}^{S T}(\omega, z) \rightarrow 0$ as $p \rightarrow \infty$. 


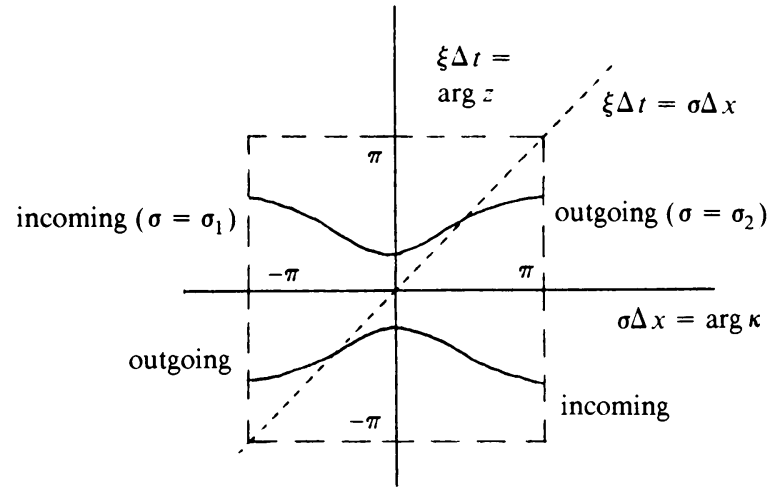

FIGURE 5.1

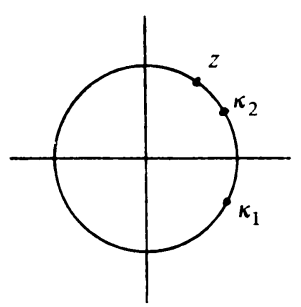

FIGURE 5.2

Proof of Proposition 5.2. Figure 5.1 shows a cross section of the dispersion relation for fixed $\omega$. A typical positioning of $z, \kappa_{1}, \kappa_{2}$ on the unit circle is indicated in Figure 5.2. It is clear that $\left|z-\kappa_{2}\right|<\left|z-\kappa_{1}\right|$ except when $\kappa_{1}$ and $\kappa_{2}$ coincide, i.e., when $\kappa_{1}=\kappa_{2}=1$ or $\kappa_{1}=\kappa_{2}=-1$. A comparison with (5.3) gives the desired conclusion.

The next proposition applies to waves that are resolved well by the grid. The proof is similar to that of Proposition 4.3 (but simpler) and is omitted.

Proposition 5.3. The reflection coefficient for space-time extrapolation of order $p$ satisfies

$$
R_{p}^{S T}(\omega, z)=-\left(\frac{\lambda-\cos \theta}{\lambda+\cos \theta}\right)^{p}+O(\xi \Delta t)
$$

where $\theta$ is the angle of incidence and $\lambda=\Delta t / \Delta x$.

The reflection is essentially zero when $\cos \theta=\lambda$. This can be described as follows. The reflection coefficient (5.3) is exactly zero if $z=\kappa_{2}$; when $|z|=\left|\kappa_{2}\right|=1$, this means $\xi \Delta t=\sigma_{2} \Delta x$, or $\sigma_{2} / \xi=\Delta t / \Delta x=\lambda$. This includes certain waves whose frequencies are quite high (see Figure 5.1). For waves that are resolved well by the grid, $\sigma_{2} / \xi \approx \cos \theta$.

An alternate approach is to consider surfaces of constant phase (in $x, y, t$ ) for outgoing plane waves. The points on the stencil of (5.1) lie on the same surface when the angle of incidence is $\pm \cos ^{-1} \lambda$. In this case, (5.1) is satisfied exactly, and there is perfect absorption.

Proposition 5.4. Space-time extrapolation of order $p$ is consistent with the analytical boundary condition

$$
\left(\lambda \frac{\partial}{\partial t}-\frac{\partial}{\partial x}\right)^{p} u=0
$$

Proof. For example, use the "forward Euler" operator

$$
\lambda\left(\frac{I-Z^{-1}}{\Delta t}\right)-Z^{-1}\left(\frac{K-I}{\Delta x}\right)
$$

or the "box scheme" operator

$$
\lambda\left(\frac{I-Z^{-1}}{\Delta t}\right)\left(\frac{I+K}{2}\right)-\left(\frac{I+Z^{-1}}{2}\right)\left(\frac{K-I}{\Delta x}\right) .
$$


6. Numerical Computations. In this section we present the results of some numerical computations involving the boundary conditions that were discussed in the preceding two sections. We obtain solutions of the wave equation on the half-space $x>0$ corresponding to various choices of initial data and boundary conditions. The results are then compared with the solutions that would be obtained if the boundary $x=0$ were not present.

The half-space solutions are obtained by computations on the spatial domain,

$$
\Omega_{1}=\{(x, y): 0<x<2,-2<y<2\} .
$$

The solutions are analyzed only on the smaller domain,

$$
\Omega_{2}=\{(x, y): 0<x<1,-1.5<y<1.5\}
$$

(see Figure 6.1). The initial data used here have compact support in $\Omega_{2}$. The boundaries of $\Omega_{1}$ are chosen so that reflections from the top, bottom, and right boundaries of $\Omega_{1}$ are not able to reach $\Omega_{2}$ during the time interval on which solutions are computed. The solutions on $\Omega_{2}$ thus coincide with true "half-space" solutions.

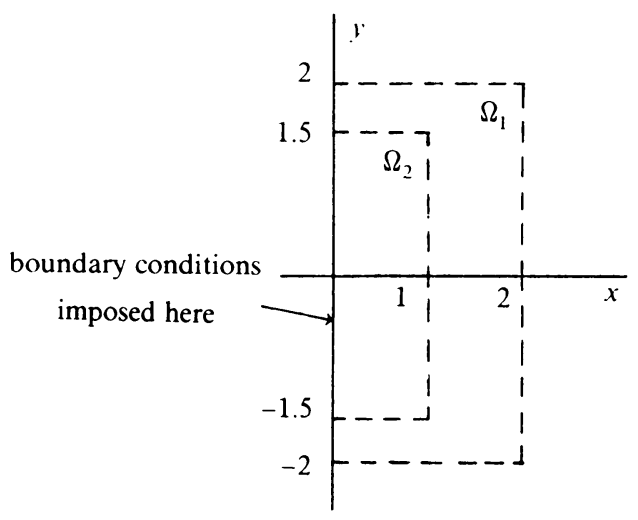

Figure 6.1

For each choice of initial data we also perform a computation on the larger domain $\{(x, y):-1<x<2,-2<y<2\}$. The restriction of this solution to $\Omega_{2}$ then gives the "free-space" solution corresponding to zero reflection. This will be used to compute reflected errors.

In the first set of tests we compare the following second-order methods. Each of these gives a smaller reflection than its first-order counterpart.

(a) Second-order averaging. This is the method (4.1) for $p=2$, and it can be written as

$$
u_{0, m}^{n+1}=\frac{1}{9}\left[\left(6 K-K^{2}\right)+Z^{-1}\left(6+4 K-2 K^{2}\right)-Z^{-2}\left(I+2 K+K^{2}\right)\right] u_{0, m}^{n+1} .
$$

Here $K$ and $Z$ are the shift operators defined in (3.3). Proposition 4.4 implies that (6.1) is consistent with

$$
\left(\lambda \frac{\partial}{\partial t}-\frac{\partial}{\partial x}\right)^{2} u=0
$$


(b) Second-order space-time extrapolation. Here we have

$$
u_{0, m}^{n+1}=2 u_{1, m}^{n}-u_{2, m}^{n-1}
$$

(see (5.1), (5.2)). This method is consistent with (6.2) (see Proposition 5.4).

(c) The second-order condition of Engquist and Majda. Here we have

$$
u_{x t}-u_{t t}+\frac{1}{2} u_{y y}=0
$$

(see [3]). We use the discretization

$$
D_{0}^{t} D_{+}^{x} u_{0, m}^{n}-\frac{1}{2} D_{+}^{t} D_{-}^{t}\left(u_{0, m}^{n}+u_{1, m}^{n}\right)+\frac{1}{4} D_{+}^{y} D_{-}^{y}\left(u_{0, m}^{n-1}+u_{1, m}^{n+1}\right)=0
$$

given in [3]. Here $D_{0}, D_{+}$, and $D_{-}$are the usual centered-, forward-, and backwarddifference operators. Superscripts denote the variables in which the differences are taken.

(d) The boundary condition (7.4) for $p=2$.

$$
\left(\frac{\partial}{\partial t}-\frac{\partial}{\partial x}\right)^{2} u=0
$$

In Proposition 7.2 this boundary condition is shown to be equivalent to (6.4). Here we approximate $\partial / \partial t-\partial / \partial x$ by

$$
\frac{I-Z^{-1}}{\Delta t}-\left(\frac{I+Z^{-1}}{2}\right)\left(\frac{K-I}{\Delta x}\right)
$$

This operator is squared to give an approximation to (6.6). The formula (6.7) is an analogue of the discretization used in Proposition 4.4. The boundary condition (6.6), (6.7) is included here mainly because its third-order analogue will be used in Tests 4 and 5 .

For later reference, we note the angles of incidence for which the above boundary conditions do their best absorption. The analytical condition of Engquist and Majda ((c) and (d)) is perfectly absorbing at normal incidence. This can be determined from their analysis in [3] and [4] or from Propositions 7.1 and 7.2 in the present paper. The averaging and space-time extrapolation methods are consistent with analytical conditions that are perfectly absorbing at angle $\cos ^{-1} \lambda$, where $\lambda$ is the mesh ratio (see (6.2) and Section 7). In the present tests, $\lambda=.625$ and $\cos ^{-1} \lambda=51.32$ degrees.

The computations were performed on a uniform grid for which $\Delta x=\Delta y=1 / 25$. In each test of each boundary condition we compare the $L^{2}$-norm of the reflected error in $\Omega_{2}$ (at various times) to the $L^{2}$-norm of the initial data. These ratios are expressed as percentages in the tables appearing below.

In Test 1 we use the initial conditions

$$
\begin{aligned}
& \text { (a) } u(x, y, 0)= \begin{cases}e^{-30 r^{2}}, & r<.45 \\
0, & r>.45\end{cases} \\
& \text { (b) } u_{t}(x, y, 0)=0,
\end{aligned}
$$

where $r^{2}=(x-.5)^{2}+y^{2}$. The Fourier transform of the Gaussian is a Gaussian centered about the zero frequency. The wave motion thus consists of Fourier modes associated with all possible directions of propagation (see Figure 2.1(b)), so a wide range of angles of incidence is present. 
The results of the computations are given in Table 6.1. At the earlier times, the Engquist-Majda condition (c) gives a smaller reflected error than do the averaging and space-time extrapolation methods. However, at later times, the averaging method gives a smaller reflected error. For the larger times, the space-time extrapolation is almost as good as condition (c).

The above behavior makes intuitive sense. The Engquist-Majda condition works better than the others for waves travelling near normal incidence. These waves reach the boundary earlier than other waves, and their reflections propagate back into the interior most rapidly. Therefore, for short times, the normally incident waves should matter most, and for such times boundary condition (c) should work better than conditions (a) and (b). However, as $t$ increases, the larger angles of incidence begin to matter. For these, the boundary conditions (a) and (b) should be more effective. The effects on the accumulated errors are evident in Table 6.1. Analogous comparisons can be made with boundary condition (d).

The space-time extrapolation method gives the largest reflected error in this particular test. However, this boundary condition may be of practical value because of its simplicity; compare (6.3) with (6.1) and (6.5).

The next two tests illustrate the behavior of the various boundary conditions at specific angles of incidence. In Test 2 we use initial conditions that produce a wave travelling at normal incidence. This is accomplished by multiplying (6.8)(a) by the wave form

$$
\cos [j(\sigma \Delta x)+m(\omega \Delta y)+n(\xi \Delta t)]
$$

where, in this case, $\sigma \Delta x=0.8, \omega \Delta y=0$, and $\xi \Delta t>0$. The dispersion relation (2.5) admits two values of $\xi \Delta t$ for each pair $(\sigma \Delta x, \omega \Delta y)$, and here we use the positive value of $\xi \Delta t$. The Fourier transform of the initial data is a Gaussian centered about the frequencies associated with (6.9). The corresponding group velocity is normal to the boundary and points out of the spatial domain (see Figure 2.2). The values of the wave packet at times $t=0$ and $t=\Delta t$ are used as initial values for the difference equation.

The results of the computations are given in Table 6.2. The boundary conditions (c) and (d) perform better than the others, as expected.

Test 3 involves a wave packet travelling at approximately 45 degrees incidence. Here we multiply a Gaussian centered at $(.5,-.5)$ by a cosine wave for which $\sigma \Delta x=0.8, \omega \Delta y=-0.8$, and $\xi \Delta t>0$. This produces a wave packet that travels toward $(0,0)$ (see Figure 2.2). In this case, boundary conditions (a) and (b) are more effective than the others; see Table 6.3.

Tests 2 and 3 suggest that if one has a priori information about the directions from which the waves are approaching the boundary, then one should adjust the boundary condition to the appropriate angle of incidence. This issue will be discussed in Sections 7 and 8.

We conclude with two tests that illustrate the effects of the generalized eigenvalue corresponding to the zero frequency. Here we use the third-order analogues of boundary conditions (a), (b), and (d). The last of these is discretized by forming the third power of (6.7). We do not use the third-order analogue of (6.4) given in [3]. 
This boundary condition is equivalent to (7.4) for $p=3$. However, it includes a term $u_{y y t}$, which appears to require an implicit difference approximation. The third-order methods (a), (b) have smaller reflection coefficients than their second-order counterparts. A similar statement holds for the analytical condition in (d) (see Proposition 7.1).

\section{TABLE 6.1}

Test 1 (Wide range of angles of incidence). Percent reflection for the following second-order methods: (a) averaging; (b) $S-T$ extrapolation; (c) Engquist-Majda; (d) $(\partial / \partial t-\partial / \partial x)^{2} u=0$.

Time

Boundary condition

$\begin{array}{rllll} & (\mathrm{a}) & (\mathrm{b}) & (\mathrm{c}) & \text { (d) } \\ .50 & 2.1 & 2.9 & 0.16 & 0.36 \\ .75 & 2.0 & 3.2 & 0.88 & 0.80 \\ 1.00 & 2.2 & 3.6 & 1.9 & 1.3 \\ 1.25 & 2.5 & 4.1 & 3.0 & 2.3 \\ 1.50 & 2.5 & 4.2 & 3.8 & 3.1 \\ 1.75 & 2.0 & 4.1 & 3.8 & 3.3\end{array}$

TABLE 6.2

Test 2 (Normal incidence). Same boundary conditions as in Test 1 .

Time Boundary condition

$\begin{array}{rllll} & (\mathrm{a}) & \text { (b) } & \text { (c) } & \text { (d) } \\ .50 & 7.3 & 4.7 & 1.8 & 3.0 \\ .75 & 8.4 & 5.9 & 2.7 & 4.7 \\ 1.00 & 8.4 & 5.9 & 2.9 & 4.9 \\ 1.25 & 8.4 & 5.9 & 2.9 & 4.9 \\ 1.50 & 7.5 & 5.0 & 2.8 & 4.7 \\ 1.75 & 2.8 & 1.6 & 1.8 & 2.9\end{array}$

TABLE 6.3

Test 3 (45 degrees incidence). Same boundary conditions as in Test 1 .

Time

$\begin{array}{rr} & (\mathrm{a}) \\ .50 & 2.7 \\ .75 & 4.7 \\ 1.00 & 5.3 \\ 1.25 & 5.6 \\ 1.50 & 5.7 \\ 1.75 & 5.5\end{array}$

Boundary condition

$\begin{array}{llrr}\text { (a) } & \text { (b) } & \text { (c) } & \text { (d) } \\ 2.7 & 1.4 & 3.5 & 1.5 \\ 4.7 & 2.1 & 9.5 & 5.2 \\ 5.3 & 2.3 & 13.6 & 8.7 \\ 5.6 & 2.5 & 14.9 & 10.0 \\ 5.7 & 2.6 & 15.4 & 10.5 \\ 5.5 & 2.5 & 15.6 & 10.7\end{array}$


TABLE 6.4

Test 4 (Effects of generalized eigenvalue. Same initial data as in Test 1). Percent reflection for: (a) 3rd order averaging; (b) $3 r d$ order $S-T ;$ (d) $(\partial / \partial t-\partial / \partial x)^{3} u=0$.

Time Boundary condition

$\begin{array}{rrrr} & \text { (a) } & \text { (b) } & \text { (d) } \\ .50 & 8.9 & 8.5 & 7.5 \\ .75 & 19.0 & 18.5 & 16.6 \\ 1.00 & 32.9 & 32.2 & 29.1 \\ 1.25 & 50.5 & 49.7 & 44.9 \\ 1.50 & 71.8 & 70.7 & 64.0 \\ 1.75 & 96.4 & 95.1 & 86.1\end{array}$

TABLE 6.5

Test 5 (Effects of generalized eigenvalue. Same initial data as in Test 2). Same boundary conditions as in Test 4.

Time

Boundary condition

$\begin{array}{rrrl} & \text { (a) } & \text { (b) } & \text { (d) } \\ .50 & 2.3 & 8.3 & 0.95 \\ .75 & 2.6 & 17.8 & 1.3 \\ 1.00 & 2.6 & 30.6 & 1.4 \\ 1.25 & 2.7 & 46.9 & 1.7 \\ 1.50 & 2.2 & 66.3 & 2.1 \\ 1.75 & 1.6 & 88.8 & 2.4\end{array}$

In the first of these tests (Test 4) we use the same initial data as in Test 1 . The Fourier transform of the initial data is essentially a Gaussian centered about the zero frequency. The results of the computations are given in Table 6.4. Each method produces a reflected error which is substantially larger than that produced by its second-order counterpart in Test 1. Additional tests with space-time extrapolation show that the amount of reflection for this method increases as the order increases. Similar effects have appeared in some computations involving a simple first-order hyperbolic system with similar initial data.

In the next test (Test 5) we use the same initial data as in Test 2. In this case the data consist mostly of higher frequencies. The results are given in Table 6.5. Boundary conditions (a) and (d) give smaller reflections than their second-order counterparts in Test 2, but space-time extrapolation shows a behavior like that in Test 4. An additional test with fourth-order space-time extrapolation yields an even higher reflection.

As noted in Section 3, the generalized eigenvalue can cause mild instabilities consisting of waves radiating spontaneously into the interior from the boundary. In Test 4 the data in the problem are concentrated about the troublesome frequency, and the instability is triggered in all of the methods. In Test 5 the data are concentrated away from the troublesome frequency, and an instability is stimulated only in method (b). 
Complete stability analyses of the discrete conditions (a) and (b) are given in Sections 4 and 5 , respectively. The only flaws in these boundary conditions correspond to the zero frequency. Similar statements hold for the corresponding analytical conditions and the analytical condition in (d) (see Proposition 7.3).

7. Generalization to Arbitrary Angles of Incidence. The discrete boundary conditions discussed in Sections 4 and 5 are consistent with analytical boundary conditions of the form

$$
\left(\lambda \frac{\partial}{\partial t}-\frac{\partial}{\partial x}\right)^{p} u=0
$$

The mesh ratio $\lambda$ is the cosine of the angle of best absorption. This suggests that one might use

$$
\left((\cos \alpha) \frac{\partial}{\partial t}-\frac{\partial}{\partial x}\right) u=0
$$

to annihilate waves moving at angle of incidence $\pm \alpha$, and that the higher-order version

$$
\left(\prod_{j=1}^{p}\left(\left(\cos \alpha_{j}\right) \frac{\partial}{\partial t}-\frac{\partial}{\partial x}\right)\right) u=0
$$

might be perfectly absorbing at angles $\pm \alpha_{1}, \ldots, \pm \alpha_{p}$. (Here $\left|\alpha_{j}\right|<\pi / 2$ for all $j$.) In this section we begin to analyze boundary conditions of the form (7.1), (7.2). From now on, we mainly consider the analytical problem rather than discrete approximations.

In the present section we discuss reflection coefficients and stability properties of (7.1) and (7.2), and we describe connections between these boundary conditions and those of Engquist and Majda [3], [4] and Bayliss and Turkel [1]. In Section 8 we discuss some practical matters relating to the implementation of (7.1), (7.2). In Section 9 we describe a useful factorization of various absorbing boundary conditions into the form (7.2).

The boundary conditions (7.1) and (7.2) have the following interpretation. Consider a plane wave of the form

$$
u(x, t)=f(x \cos \alpha+y \sin \alpha+t),
$$

where $f$ is some function. This wave moves out of the spatial region $x>0$ at angle of incidence $\alpha$. It also satisfies (7.1). The boundary condition (7.1) can thus be regarded as a compatibility condition for waves of the above form. In particular, the boundary condition $(\partial / \partial t-\partial / \partial x) u=0$ is compatible with outgoing waves moving at normal incidence (see Proposition 7.2). Similarly, a linear combination of plane waves moving outward at angles $\pm \alpha_{1}, \ldots, \pm \alpha_{p}$ would satisfy exactly the higher-order version (7.2).

Proposition 7.1. The reflection coefficient for (7.2) is

$$
-\prod_{j=1}^{p}\left(\frac{\cos \alpha_{j}-\cos \theta}{\cos \alpha_{j}+\cos \theta}\right),
$$

where $\theta$ is the angle of incidence. 
Proof. Insert into condition (7.2) a linear combination of an incoming wave $\exp \left(i \sigma_{1} x+i \omega y+i \xi t\right)$ and an outgoing wave $\exp \left(i \sigma_{2} x+i \omega y+i \xi t\right)$ to get

$$
R=-\prod_{j=1}^{p}\left(\frac{\left.\left(\cos \alpha_{j}\right) i \xi-i \sigma_{2}\right)}{\left.\left(\cos \alpha_{j}\right) i \xi-i \sigma_{1}\right)}\right) .
$$

Now use $\sigma_{2} / \xi=\cos \theta, \sigma_{1} / \xi=-\cos \theta$ (see Figure 4.3).

The absolute value of each factor in (7.3) is less than 1 , except when $\theta=\pi / 2$. This exception is of no consequence, since this case corresponds to tangential incidence. The corresponding modes do not propagate into the interior.

We next relate the general formulation (7.2) to the boundary conditions derived by Engquist and Majda [3], [4] for the wave equation. Their derivation is quite different from that implied by the next proposition (cf. Section 9).

Proposition 7.2. The pth-order boundary condition of Engquist and Majda is equivalent to

$$
\left(\frac{\partial}{\partial t}-\frac{\partial}{\partial x}\right)^{p} u=0
$$

i.e., $\alpha_{j}=0$ for all $j$.

Proof. When $p=1$, Eq. (7.4) is identical to their first-order boundary condition. When $p=2,(7.4)$ is

$$
u_{t t}-2 u_{x t}+u_{x x}=0 .
$$

But (1.1) says $u_{x x}=u_{t t}-u_{y y}$, so the boundary condition can be written in the form

$$
2 u_{t t}-2 u_{x t}-u_{y y}=0 \text {. }
$$

This is equivalent to Eq. (2) in [3]. A similar treatment of the case $p=3$ yields Eq. (3) in [3].

For the general case, use the recursion relation

$$
B_{p+1} u=\frac{\partial}{\partial t} B_{p} u-\frac{1}{4} \frac{\partial^{2}}{\partial y^{2}} B_{p-1} u
$$

given in Eq. (1.5) in their second paper [4]. Here $B_{p}$ is the operator used in the boundary condition of order $p$. In [4] the spatial domain is defined by $x<a=$ constant, so the signs of the $x$-derivatives should be reversed when that paper is compared with this one. An induction shows

$$
B_{p}=\frac{1}{2^{p-1}}\left(\frac{\partial}{\partial t}+\frac{\partial}{\partial x}\right)^{p}
$$

The interior equation $u_{t}=u_{x x}+u_{y y}$ is used during this induction. When the necessary notational change is made, we get (7.4).

The reflection coefficient for (7.4) is

$$
-\left(\frac{1-\cos \theta}{1+\cos \theta}\right)^{p}
$$

(cf. (7.3)). This has a zero of multiplicity $2 p$ at $\theta=0$, whereas the coefficient for (7.2) has zeros at $\pm \alpha_{1}, \ldots, \pm \alpha_{p}$. The general form (7.2) thus enables one to spread out the zeros and thereby broaden the range of angles where the reflection coefficient is small. The results in Test 1 in Section 6 suggest the value of this approach. Also see Section 8. 
Proposition 7.2 suggests a connection between the boundary conditions in [3], [4] and those of Bayliss and Turkel [1]. The latter conditions are designed for computations on exterior domains and use compositions of operators of the form $\partial / \partial t+$ $\partial / \partial r+$ constant $/ r$, where $r$ is a radial distance. The coefficients of $1 / r$ are chosen so that the boundary condition annihilates as many terms as possible in asymptotic expansions of outgoing solutions. The expansions are valid as $r \rightarrow \infty$.

We next discuss stability properties of the boundary conditions (7.1) and (7.2). A standard energy argument shows that when the first-order condition (7.1) is used, the energy seminorm

$$
\iint_{\Omega}\left(\left|u_{t}\right|^{2}+|\nabla u|^{2}\right) d x d y
$$

is nonincreasing in time. We omit the details.

For the more general case (7.2) we use a stability criterion which is essentially that used in the "normal mode analysis" developed by Kreiss and others (e.g., [10]) for studying well-posedness of initial-boundary value problems for first-order hyperbolic systems (also see [8]). This criterion is an analogue of the criterion (3.7) used earlier for difference approximations.

The criterion can be described as follows. Denote a homogeneous boundary condition involving $x, t$ derivatives by

$$
B(\partial / \partial x, \partial / \partial t) u=0,
$$

where $B$ is a polynomial in two variables. Here the symbol " $B$ " has a different meaning than it did in Section 3. Consider solutions of (1.1) that have the form

$$
e^{\gamma x+i \omega y+s t}
$$

where $\gamma$ and $s$ are complex numbers, $\operatorname{Re} s \geqslant 0$, and $\operatorname{Re} \gamma \leqslant 0$. (The dual variables must satisfy $s^{2}=\gamma^{2}+(i \omega)^{2}$.) The stability criterion is

$$
B(\gamma, s) \neq 0 \text { for } \operatorname{Re} s \geqslant 0 \text {. }
$$

The value of $\gamma$ for $\operatorname{Re} s=0$ is defined to be the limit of values of $\gamma$ corresponding to $\operatorname{Re} s>0$.

Consider two cases of (7.7):

(1) Suppose that $\operatorname{Re} s>0, \operatorname{Re} \gamma<0$, and (7.6) satisfies the boundary condition (7.5). The related modes $\exp [c(\gamma x+i \omega y+s t)]$ are also solutions of the initialboundary value problem, for any $c$. (This requires that the various terms in the boundary condition have the same total degree, as is the case for (7.2).) As $c \rightarrow \infty$ these solutions grow exponentially in $t$ at higher and higher rates, but they still retain finite norm in $x$. The initial-boundary value problem would then be strongly ill-posed (see [8] or [10]). The condition (7.7) guarantees that these modes are excluded.

(2) Next consider the limit $\operatorname{Re} s \rightarrow 0$, i.e., $s \rightarrow i \xi$. If $\operatorname{Re} \gamma \rightarrow 0$ also (i.e., $\gamma \rightarrow i \sigma$ ), then the limiting value must be $i \sigma_{1}$ (incoming) rather than $i \sigma_{2}$ (outgoing) (see [8]). As in Section 3, these modes must be prevented from satisfying the boundary condition by themselves. Equivalently, condition (7.7) enables one to solve for incoming modes in terms of outgoing modes, since $B\left(i \sigma_{1}, i \xi\right)$ is the denominator in the reflection coefficient. (Also see [8].) The condition (7.7) also excludes evanescent modes for which $\operatorname{Re} \gamma<0$. 
Proposition 7.3. If $\left|\alpha_{j}\right|<\pi / 2$ for all $j$, then the boundary conditions (7.2) satisfy the criterion (7.7), with the exception that $B(0,0)=0$.

The exceptional case means that we again have a generalized eigenvalue corresponding to the zero frequency. In the notation used for the discrete problem, this was the case $\kappa=z=1$.

Proof of Proposition 7.3. Let $B(\partial / \partial x, \partial / \partial t) u=0$ denote the boundary condition (7.2). Then,

$$
B(\gamma, s)=\prod_{j=1}^{p}\left(\left(\cos \alpha_{j}\right) s-\gamma\right) .
$$

Here $\cos \alpha_{j}>0$ for all $j$.

If $\operatorname{Re} s>0$ and $\operatorname{Re} \gamma<0$, then each of the factors is nonzero.

Next consider the case $\operatorname{Re} s=0$. If $\operatorname{Re} \gamma<0$, then again each factor is nonzero. If $\operatorname{Re} \gamma=0$, then $\gamma=i \sigma_{1}$ (incoming), and a typical factor can be written as $\left(\cos \alpha_{j}\right) i \xi$ $-i \sigma_{1}$. But $\xi$ and $\sigma_{1}$ have opposite signs (see Figure 2.1(b)), so each factor in (7.8) is nonzero except if $\xi=\sigma_{1}=0$. This gives the desired conclusion.

The proof shows that if a boundary condition can be factored into a form like (7.2), then its stability analysis reduces to an analysis of one-dimensional first-order operators.

8. Practical Considerations. Here we discuss various matters related to the implementation of the boundary conditions (7.2) and the choice of suitable angles $\alpha_{j}$. Consider the second-order case

$$
\left(\left(\cos \alpha_{1}\right) \frac{\partial}{\partial t}-\frac{\partial}{\partial x}\right)\left(\left(\cos \alpha_{2}\right) \frac{\partial}{\partial t}-\frac{\partial}{\partial x}\right) u=0 .
$$

One way to implement (8.1) would be to multiply the two operators in (8.1) and obtain a linear combination of $u_{t t}, u_{x t}$, and $u_{x x}$. If the boundary condition is used in this form, it would be necessary to approximate $u_{x x}$ with one-sided differences. It might be difficult to obtain a stable difference method by this approach, so one could use the interior equation $u_{x x}=u_{t t}-u_{y y}$ to write the boundary condition in terms of $u_{t t}, u_{x t}$, and $u_{y y}$. This would be similar to a process outlined in the proof of Proposition 7.2.

An alternate approach is to approximate each factor in (8.1) with a one-dimensional, first-order difference operator. Then use the composition of these operators to approximate (8.1). This was done for boundary condition (d) in Section 6. Such an approach would have the following advantages:

(1) The stencil of the boundary condition would be one-dimensional, even for a higher-order boundary condition. This means that it would not be necessary to adopt any special boundary procedures near a corner (unlike in the case of a boundary condition that involves tangential derivatives). In fact, if the interior scheme (2.4) were used with a one-dimensional boundary condition, then the corner points would never be involved in the computation.

(2) The analysis of stability would be simplified. In general, stability analyses can be quite complicated, but in the present case, the analysis would reduce to a study of first-order factors having one-dimensional stencils. The situation would resemble that in the proof of Proposition 7.3. This approach will be used in [9] to give a general stability analysis of approximations of (7.2). 
Next, consider the choice of the angles $\alpha_{1}, \alpha_{2}$ appearing in (8.1). In general, the optimal $\alpha_{1}, \alpha_{2}$ would depend on the problem being considered. For example, suppose that a computation is performed on a rectangular domain and that the initial data have compact support in the domain. The closer the support is to any given boundary segment, then the broader the range of possible angles of incidence, and the broader the range from which the optimal $\alpha_{1}, \alpha_{2}$ are to be chosen. In any given situation, it may be necessary to determine these angles experimentally.

The boundary condition can also be adjusted to take advantage of a priori information about the solution. For example, if the wave motion arises from a localized source, then the angle of incidence at each boundary point is fairly well-defined, and at each point, $\alpha_{1}$ and $\alpha_{2}$ can be chosen accordingly.

9. A Factorization Theorem. In this section we describe a factorization process that provides a fairly general characterization of absorbing boundary conditions.

We first outline a procedure that has been used by other authors to derive such conditions. Figure 2.1(b) and the dispersion relation (2.2) imply that outgoing waves are characterized by

$$
\sigma_{2}(\omega, \xi)=\xi\left(1-\omega^{2} / \xi^{2}\right)^{1 / 2}
$$

(The negative square root would describe incoming waves.) If $r(\omega / \xi)$ is a rational approximation to the square root in $(9.1)$, then

$$
\sigma_{2}(\omega, \xi) \approx \xi r(\omega / \xi) .
$$

Now, clear denominators in (9.2) and move everything to one side. The resulting polynomial is the symbol of a differential operator that approximately annihilates outgoing waves and thus yields an absorbing boundary condition. The form (9.2) implies that all terms in the operator have the same total degree.

This procedure was introduced by Engquist and Majda ([3], [4]), who used Padé approximations. Wagatha [15] later used least-squares approximations. Trefethen and Halpern [14] have made a general study of rational approximations, with applications to absorbing boundary conditions and to one-way wave equations.

The square root in (9.1) is an even function. If $r(\omega / \xi)$ is also even, and if the numerator and denominator do not have any factors in common, then all powers of $\omega$ must have even order. This means that all of the $y$-derivatives in the boundary condition have even order, and the interior equation (1.1), $u_{t t}=u_{x x}+u_{y y}$, can be used to eliminate these derivatives. From now on we will assume that this is the case. (However, the theory in [14] includes the possibility of nonsymmetric approximations, and in applications to one-way wave equations it is not possible to use (1.1) to perform the last step.)

Under the above assumption, the boundary condition has the form

$$
P(\partial / \partial x, \partial / \partial t) u=0,
$$

where $P$ is a real polynomial in which the terms have the same total degree. The homogeneity means that $P$ can be factored into the form

$$
\prod_{j} Q_{j}(\partial / \partial x, \partial / \partial t)
$$

where each $Q_{j}$ is either linear or real irreducible quadratic. Our aim is to characterize the factors $Q_{j}$. 
The factorization of the Engquist-Majda conditions is given in Proposition 7.2. The second-order condition of Wagatha [15] is

$$
\frac{21 \pi}{64} u_{t t}-u_{x t}-\frac{15 \pi}{64} u_{y y}=0
$$

which factors into the form (7.2), with $\alpha_{1} \doteq 22.14$ and $\alpha_{2} \doteq 64.42$ degrees.

Proposition 9.1. The boundary condition (9.3), (9.4) either

(1) factors into the form (7.2), or

(2) violates the stability criterion (7.7), or

(3) is not optimal, in the sense that it is possible to modify the coefficients in at least one of the $Q_{j}$ so as to reduce the magnitude of the reflection coefficient.

The factored form (7.2) does a great deal to simplify stability analyses (see Proposition 7.3) and numerical implementation (see Section 8).

Proof of Proposition 9.1. The stability criterion (7.7) is equivalent to $Q_{j}(\gamma, s) \neq 0$ for all $j$, and the reflection coefficient is

$$
-\prod_{j} \frac{Q_{j}\left(i \sigma_{2}, i \xi\right)}{Q_{j}\left(i \sigma_{1}, i \xi\right)}
$$

(cf. Proposition 7.1). We can thus analyze the factors individually. A factor $Q$ must have the form

(I) $b \partial / \partial t-\partial / \partial x$, where $b$ is real, or

(II) irreducible real quadratic.

First consider case (I).

If $0<b \leqslant 1$, then $Q$ has the form of a factor in (7.2), with $\cos \alpha=b$.

If $-1 \leqslant b<0$, then $Q$ allows incoming waves at angle $\cos ^{-1} b$. This is a form of instability.

The case $b<-1$ corresponds to exponential instability; use $s=|\omega| /\left(b^{2}-1\right)^{1 / 2}$ and $\gamma=s b$.

If $b>1$ there is no instability. However, the corresponding contribution to the reflection coefficient is

$$
R_{Q}=\frac{b-\left(\sigma_{2} / \xi\right)}{b-\left(\sigma_{1} / \xi\right)} .
$$

Here $\sigma_{2} / \xi=-\sigma_{1} / \xi \geqslant 0$. See Figure 9.1, and compare with Figure 2.1(b). A small decrease in $b$ yields a decrease in $\left|R_{Q}\right|$, whenever $\sigma_{1} \neq \sigma_{2}$. Thus, $Q$ can be modified

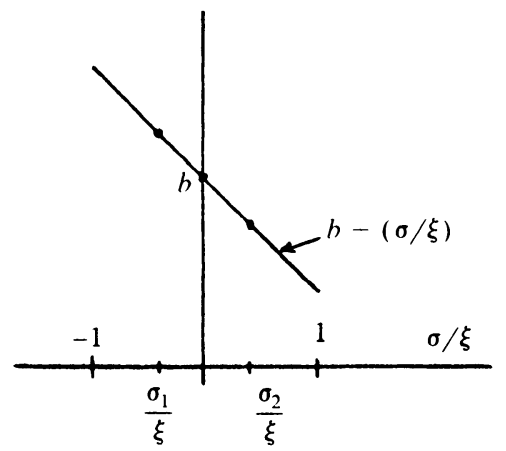

FIGURE 9.1

Case (I) for $b>1$. 
to give less reflection, and it cannot be part of an optimal boundary condition. The case $b=0$ is also not optimal.

In case (II) the quadratic polynomial $Q(\sigma / \xi, 1)$ is never zero. Without loss of generality, assume that it is positive. If the vertex of the parabola is centered or to the left of the origin, then $\left|Q\left(\sigma_{2} / \xi, 1\right) / Q\left(\sigma_{1} / \xi, 1\right)\right| \geqslant 1$. Otherwise, this ratio is less than 1 . However, it can be reduced further by bringing the parabola closer to the horizontal axis. Since this case cannot be optimal, we will not bother to analyze stability.

The factorization can be described in terms of points where the rational approximation $r$ in (9.2) equals the square root in (9.1). At any such point, $\sigma_{2}(\omega, \xi)=$ $\xi r(\omega / \xi)$. This means that the outgoing mode $\exp \left[i \sigma_{2}(\omega, \xi) x+i \omega y+i \xi t\right]$ satisfies exactly the boundary condition derived from (9.2), and perfect absorption occurs. But this case also gives a root of $P(\sigma / \xi, 1)$, which yields a factor of $P(\partial / \partial x, \partial / \partial t)$.

Acknowledgment. I thank L. N. Trefethen for useful comments on various matters related to the subject of this paper.

Department of Mathematics

Oregon State University

Corvallis, Oregon 97331

1. A. Bayliss \& E. Turkel, "Radiation boundary conditions for wave-like equations," Comm. Pure Appl. Math., v. 33, 1980, pp. 707-725.

2. R. M. BeAm, R. F. WARMing \& H. C. YeE, "Stability analysis of numerical boundary conditions and implicit difference approximations for hyperbolic equations," J. Comput. Phys., v. 48, 1982. pp. 200-222.

3. B. ENGQUiST \& A. MAJDA, "Absorbing boundary conditions for the numerical simulation of waves," Math. Comp., v. 31, 1977, pp. 629-651.

4. B. ENGQuist \& A. MAJDA, "Radiation boundary conditions for acoustic and elastic wave calculations," Comm. Pure Appl. Math., v. 32, 1979, pp. 313-357.

5. B. GustafsSon, H.- O. Kreiss \& A. SundSTROM, "Stability theory of difference approximations for mixed initial boundary value problems. II," Math. Comp., v. 26, 1972, pp. 649-686.

6. B. GuSTAFSSON \& J. Oliger, "Stable boundary approximations for implicit time discretizations for gas dynamics," SIAM J. Sci. Statist. Comput., v. 3, 1982, pp. 408-421.

7. L. HALPERN, "Absorbing boundary conditions for the discretization schemes of the one-dimensional wave equation," Math. Comp., v. 38, 1982, pp. 415-429.

8. R. L. HIGDON, "Initial-boundary value problems for linear hyperbolic systems," SIAM Rev., v. 28. 1986, pp. 177-217.

9. R. L. Higdon, "Numerical absorbing boundary conditions for the wave equation." (To appear.)

10. H.- O. Kreiss, "Initial boundary value problems for hyperbolic systems," Comm. Pure Appl. Math., v. 23, 1970, pp. 277-298.

11. D. Michelson, "Stability theory of difference approximations for multidimensional initial-boundary value problems," Math. Comp., v. 40, 1983, pp. 1-45.

12. L. N. Trefethen, "Group velocity in finite difference schemes," SIAM Rel'., v. 24, 1982, pp. $113-136$

13. L. N. TREFETHEN, "Instability of difference models for hyperbolic initial boundary value problems," Comm. Pure Appl. Math., v. 37, 1984, pp. 329-367.

14. L. N. TREFEThen \& L. HALPERN, "Well-posedness of one-way wave equations and absorbing boundary conditions." Math. Comp., v. 47, 1986, pp. 421-435.

15. L. WaGatha, "Approximation of pseudodifferential operators in absorbing boundary conditions for hyperbolic equations," Numer. Math., v. 42, 1983, pp. 51-64.

16. G. B. Whiтham, Linear and Nonlinear Waves, Wiley-Interscience, New York, 1974. 